\title{
Evaluation of Lumicyano cyanoacrylate fuming process for the development of latent fingermarks on plastic carrier bags by means of a pseudo operational comparative trial
}

\author{
Kevin J. Farrugia ${ }^{1}$, Paul Deacon ${ }^{2}$, Joanna Fraser ${ }^{1}$ \\ ${ }^{1}$ School of Science, Engineering \& Technology, Division of Computing and Forensic \\ Sciences, University of Abertay, Dundee, DD1 1HG, UK \\ ${ }^{2}$ c/o School of Science, Engineering \& Technology, Division of Computing and Forensic \\ Sciences, University of Abertay, Dundee, DD1 1HG, UK
}

\section{* Corresponding Author:}

School of Science, Engineering \& Technology

Division of Computing and Forensic Sciences

University of Abertay

Dundee DD1 1HG

United Kingdom

tel: +44 (0) 1382308689

kevin.farrugia@abertay.ac.uk 


\title{
Evaluation of Lumicyano ${ }^{\mathrm{TM}}$ cyanoacrylate fuming process for the development of latent fingermarks on plastic carrier bags by means of a pseudo operational comparative trial
}

\begin{abstract}
There are a number of studies discussing recent developments of a one-step fluorescent cyanoacrylate (superglue) process. This study carried out a pseudo operational trial to compare an example of a one-step fluorescent superglue product, Lumicyano, with the two recommended techniques for plastic carrier bags; superglue fuming followed by basic yellow 40 (BY40) dyeing and powder suspension. 100 plastic carrier bags were collected from the place of work and the items were treated as found without any fingermark deposition. The bags were split into three and treated with the three techniques and a comparable number of fingermarks was detected by each technique (average of 300 fingermarks). The items treated with Lumicyano were sequentially processed with BY40 and an additional 43 fingermarks were detected. Lumicyano appears to be a suitable technique for the development of fingermarks on plastic carrier bags and it can help save lab space and time as it does not require dyeing or drying procedures. Furthermore, contrary to other one-step cyanoacrylate products, existing superglue cabinets do not require any modification for the treatment of articles with Lumicyano. To date, there is little peer reviewed articles in the literature on trials related to Lumicyano and this study aims to contribute to fill this gap.
\end{abstract}

Keywords: Lumicyano, powder suspension, superglue, basic yellow 40, fluorescent, fingermarks, cyanoacrylate 


\section{Introduction}

The UK Home Office Centre for Applied Science and Technology (CAST) currently recommends either the use of superglue followed with basic yellow 40 (BY40) dyeing or ironbased powder suspension as the primary method for the enhancement of latent fingermarks on plastic packaging material [1-2]. This study [2] also found that the effectiveness of vacuum metal deposition (VMD) on this substrate has diminished relative to that of superglue fuming followed by BY40; however, the use of VMD may detect additional marks when used in sequence after superglue/BY40.

A new product on the forensic market, Lumicyano, combines the superglue fuming and the dyeing procedure into a one-step process offering the potential to save time and effort in the detection of latent fingermarks [3]. There are other products currently on the market that offer a one-step fluorescent cyanoacrylate fuming process such as PolyCyano by Foster and Freeman Ltd. An evaluation study of this product by Hahn and Ramotowski [4] revealed that this product is comparable to the conventional two-step fuming and staining method. This method; however, requires a modification of existing cabinets since PolyCyano is a solid powder and requires heating temperatures of up to $230^{\circ} \mathrm{C}$. The use of such high temperatures for cyanoacrylates may produce toxic hydrogen cyanide gas [5]. Other one-step fluorescent fuming products such as fuming orange and $\mathrm{CN}$ yellow also require higher temperatures for fuming evidence compared to the standard $120^{\circ} \mathrm{C}[6]$.

This pseudo operational trial aims to compare superglue/BY40, Lumicyano and iron-based powder suspension to investigate the suitability and effectiveness of each technique for the visualisation of fingermarks on plastic carrier bags. CAST [7] defines pseudo operational trials as a trial to "establish whether the results obtained in laboratory trials are replicated on articles/surfaces typical of those that may be submitted to a fingerprint laboratory, or to distinguish between closely equivalent formulations that cannot be separated in laboratory trials." Plastic carrier bags were selected as the test substrate in the trial as they cover most plastic packaging material types handled by the general public on a daily basis [1] as well as a direct comparison to previous studies [2]. 


\section{Materials and Methods}

\section{Sample preparation}

A request for plastic carrier bags was issued to work colleagues to obtain different types of bags with varying ages and fingermark donors. The maximum number of bags from each colleague was limited to 5 with random origins, use and age. An initial trial of 100 carrier bags was carried out to reflect other studies [2] and the description (e.g. colour and plastic type) for each bag was recorded. All bags were split into three equal parts and labelled A, B and $\mathrm{C}$ respectively (left to right). On bag 1 part $\mathrm{A}$ will correspond to Lumicyano, part $\mathrm{B}$ to superglue/BY40 and part C to iron-based powder suspension (figure 1). To eliminate any bias, the techniques will be rotated for each third of the bag throughout the trial - for example bag 2 part A will correspond to iron-based powder suspension, part B to Lumicyano and part $\mathrm{C}$ to superglue/BY40.

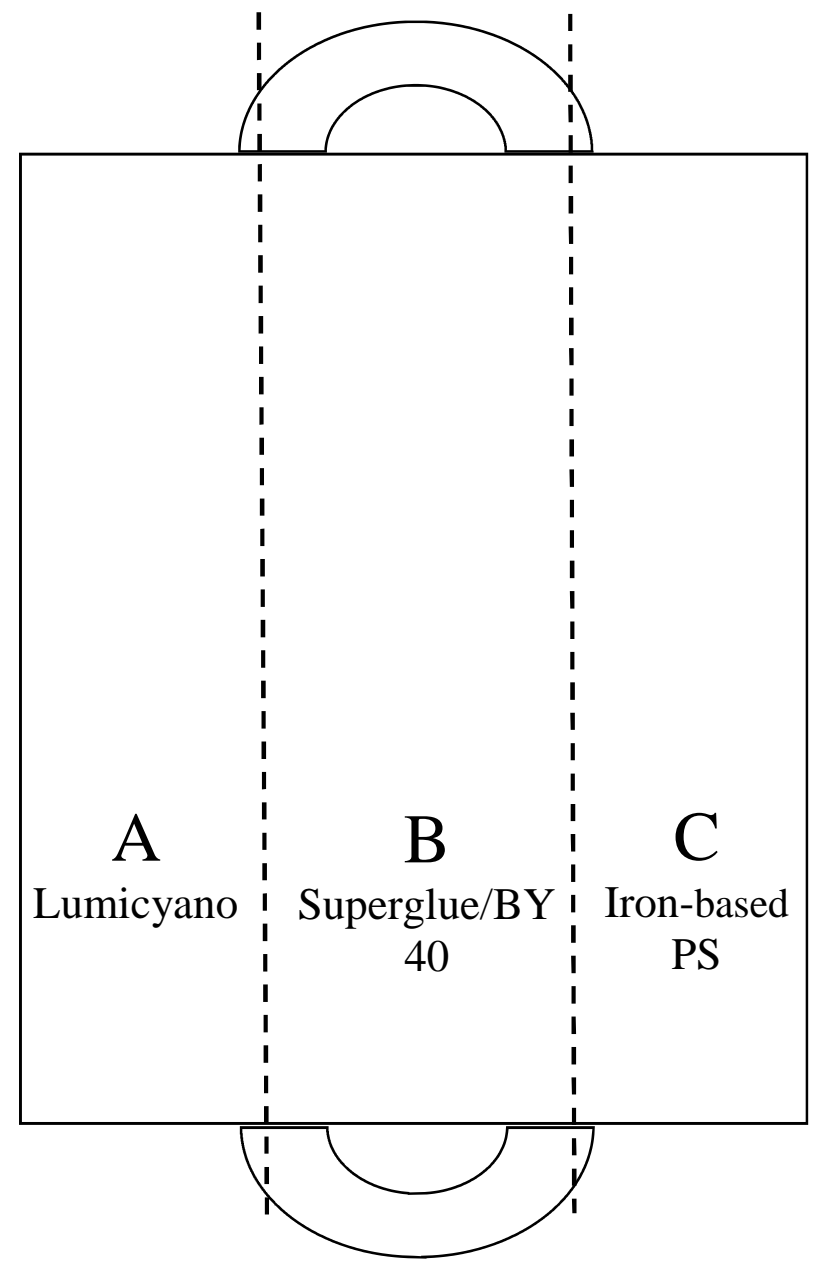

Figure 1 - Sample division for a plastic carrier bag in the study 
An Air Science (model number CA305) fuming chamber was employed with an approximate volume of about 450 litres. The chamber is fitted with a fixed temperature hot plate (internally set to $90^{\circ} \mathrm{C}$ ) and a humidifier (set to $80 \%$ ). Before the start of the trial, the correct operation of the hot plate and humidifier were verified by means of a thermocouple (Fluke 50 Series II) and a humidity meter (Fluke 971). Fluorescence examination was performed using a Mason Vactron Quaser 2000/30 and photography was carried out using a Nikon D5100 equipped with a $60 \mathrm{~mm}$ micro Nikon lens. UV examination was carried out using a UV Light Technology light source (GF UV 35W backlight torch).

\section{Superglue/BY40}

$2 \mathrm{~g}$ of superglue (CSI equipment Ltd, UK) was placed into a new foil dish and positioned on a clean support ring on a heat source of about $90^{\circ} \mathrm{C}$ in the fuming chamber. The relative humidity level within the chamber was set at $80 \%$ with a running time of 45 minutes. A cycle time of 45 minutes ensured that $99.99 \%$ of the glue had evaporated as checked by the weight difference before and after the cycle. The fuming process was followed by immersion of the items under examination in a BY40 solution for 1 minute followed by thorough rinsing under running tap water and left to dry at room temperature before fluorescence examination.

Basic yellow 40 (CSI equipment Ltd, UK) dye was prepared by dissolving $2 \mathrm{~g}$ in $1 \mathrm{~L}$ ethanol (Fisher). Fluorescence was observed using a Quaser 2000/30 by exciting with a violet/blue excitation source (band pass filter $350-469 \mathrm{~nm}$ at $1 \%$ cut-on and cut-off points respectively) and viewed with a yellow long pass $476 \mathrm{~nm}$ filter (1\% cut-on point). Other light sources may use wavelengths representing the $50 \%$ point or the peak wavelength.

\section{Lumicyano $^{T M}$}

$2 \mathrm{~g}$ of Lumicyano was placed into a new foil dish and positioned on a clean support ring on a heat source of about $90^{\circ} \mathrm{C}$ in the fuming chamber. The relative humidity level within the chamber was set at $80 \%$ with a running time of 45 minutes. A cycle time of 45 minutes ensured that $99.99 \%$ of the glue had evaporated as checked by the weight difference before and after the cycle. The manufacturers of this product state that fluorescence can be observed either under UV light $(315-340 \mathrm{~nm})$ or visible $(450-550 \mathrm{~nm})$ intense light irradiation (figure 2). After fuming, in this study, fluorescence was observed using the Quaser 2000/30 by exciting with a blue/green light (band pass filter $468-526 \mathrm{~nm}$ at $1 \%$ cut-on and cut-off points respectively) and viewed with an orange long pass $529 \mathrm{~nm}$ filter (1\% cut-on point). UV 
fluorescence was performed using UV Light Technology light source (peak excitation at $325 \mathrm{~nm}$ ) and viewed with a standard UV filter.

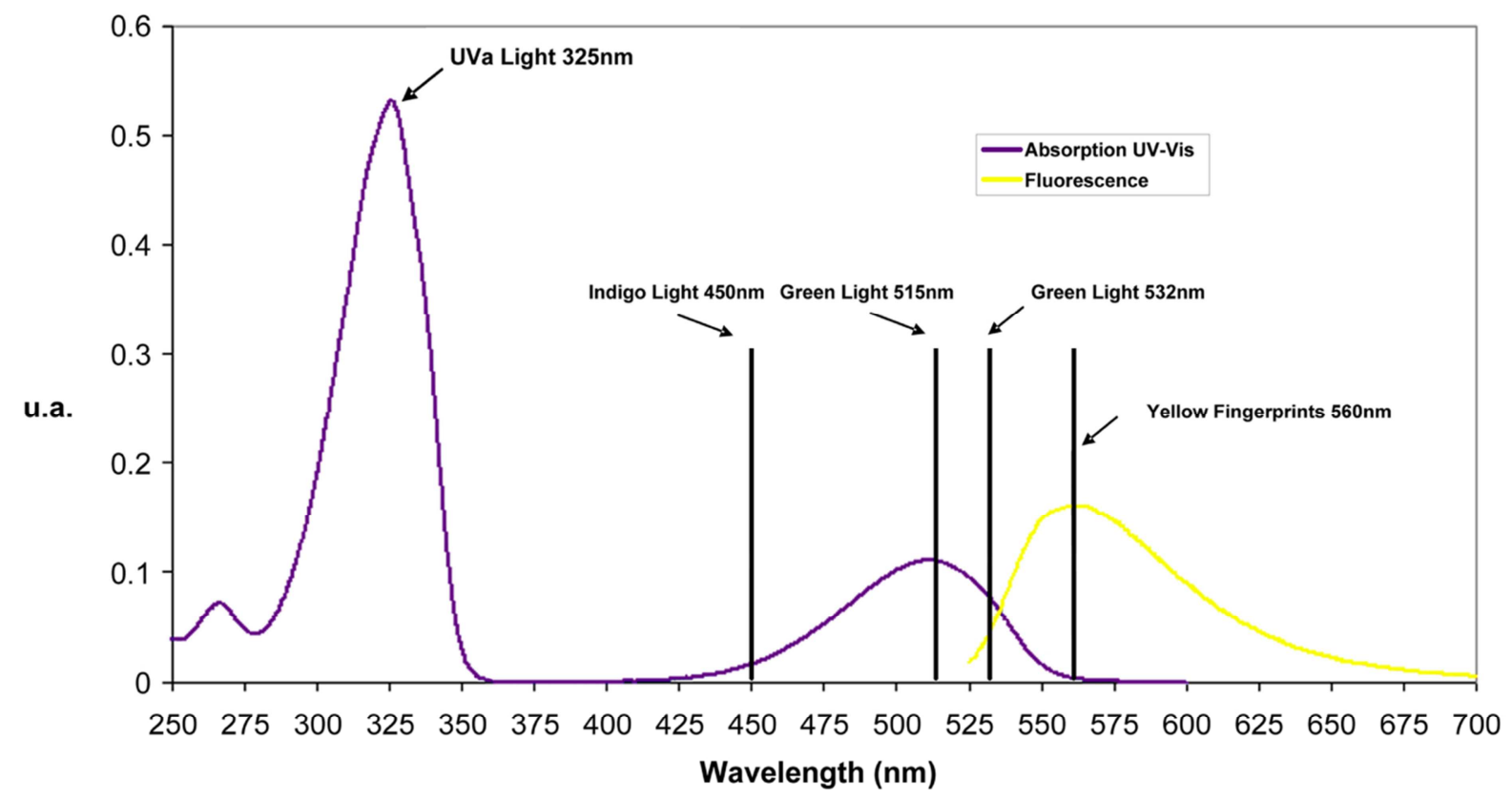

Figure 2 - Lumicyano $^{\mathrm{TM}}$ : Absorption UV-Vis and Fluorescence

\section{Iron-based black powder suspension}

Iron (II/III) oxide (20g, Fischer I/1100/53) was weighed and poured into a 100mL glass beaker. Stock detergent solution $(20 \mathrm{~mL})$ was added slowly whilst stirring with a soft squirrel hair brush until no lumps remained. The stock detergent solution was prepared by measuring Triton X100 (250mL, Acros) and adding ethylene glycol (350mL, Acros) whilst stirring slowly for 10 minutes. Distilled water $(400 \mathrm{~mL})$ was added and stirred for a further 10 minutes. The articles to be treated were wetted with tap water prior to the application of the powder suspension with a small, animal hair brush. The working suspension was left for a few seconds and then washed under slowly running, cold tap water until all the excess powder is removed from the background. The article was allowed to dry at room temperature before examination.

\section{Titanium-based white powder suspension}

For carrier bags that were black or dark coloured, a white powder suspension was employed. A commercial product WetWop was applied and rinsed as described above for black powder suspension. 
Evaluation of the number and quality of latent marks recovered by each process Any prints developed with continuous ridge detail and an area greater than $64 \mathrm{~mm}^{2}$ were counted $[2,4]$. Each of these marks were graded 'a' for good contrast or 'b' for poor contrast as well as assessed for the quality of pore and ridge detail (the presence of third level detail or not). Marks that showed signs of over-fuming were also noted.

\section{Evaluation of the stability of Lumicyano fluorescence}

A selection of fingermarks developed with Lumicyano ( $\sqrt{\text { total}})$ was investigated further for the stability of fluorescence. Photographs of these marks were taken 1 hour, 1 day and 7 days after development. Half of each sample was stored in a sealed Kraft envelope at room temperature in a cool, dry and dark cupboard and the other half left on an open bench for the same period of time. The representative samples were then re-fumed with Lumicyano followed by subsequent BY40 dyeing. 


\section{Results and Discussion}

Table 1 provides further details for the100 bags used in this study.

Table 1 - Detailed information about the plastic carrier bags used in this study

\begin{tabular}{|c|c|c|c|}
\hline Plastic bag number & Colour & Brand & Plastic type \\
\hline 1 & maroon & clothing alteration company & LDPE \\
\hline 2 & yellow & Start Fitness & LDPE \\
\hline 3 & blue & Next & LDPE \\
\hline 4 & white & Greece Duty Free & LDPE \\
\hline 5 & white & WHSmith & HDPE \\
\hline 6 & transparent & Morrisons & HDPE \\
\hline 7 & red & $\mathrm{M} \& \mathrm{~S}$ & LDPE \\
\hline 8 & black & Debenhams & LDPE \\
\hline 9 & green & Forever Fish (M\&S) & HDPE \\
\hline 10 & white & New Look & LDPE \\
\hline 11 & white & Tesco & HDPE \\
\hline 12 & transparent & Tesco & HDPE \\
\hline 13 & Orange & Dundee United & LDPE \\
\hline 14 & Green/white & Supastitch & LDPE \\
\hline 15 & blue & Debenhams & LDPE \\
\hline 16 & Yellow/blue & NL airport schiphol & LDPE \\
\hline 17 & white & Sotmid & HDPE \\
\hline 18 & white & ASDA & HDPE \\
\hline 19 & green & ELC & HDPE \\
\hline 20 & white/blue & Tesco (together for trees) & LDPE \\
\hline 21 & mutlicolour & Tesco (Bunny) & LDPE \\
\hline 22 & white & Pitlochry Festival & Cellulose \\
\hline 23 & Grey & Annika & LDPE \\
\hline 24 & white/red & Confections/Dist & LDPE \\
\hline 25 & orange & Sainsbury's & HDPE \\
\hline 26 & blue & Roche & HDPE \\
\hline 27 & white & Blood Dundee & LDPE \\
\hline 28 & white & Music Room & Cellulose \\
\hline 29 & Yellow & Hawkins Bazaar & LDPE \\
\hline 30 & green & Superdrug & LDPE \\
\hline 31 & Dark Green & $M \& S$ & LDPE \\
\hline 32 & mutlicolour & LIDL & LDPE \\
\hline 33 & White/Red & Iceland & HDPE \\
\hline 34 & white/blue & Tesco & HDPE \\
\hline 35 & Black & $\mathrm{DP}$ & LDPE \\
\hline 36 & white/red & Pound Stretcher & HDPE \\
\hline 37 & transparent/green & Clarks & LDPE \\
\hline 38 & white/red & Home Bargains & HDPE \\
\hline 39 & White & Farm Foods & HDPE \\
\hline 40 & white/blue & Gillies & LDPE \\
\hline 41 & Green & ASDA & LDPE \\
\hline 42 & multicolour & Millars & LDPE \\
\hline 43 & transparent/black & McKenzie & LDPE \\
\hline 44 & white & $\mathrm{COOK}$ & LDPE \\
\hline 45 & Black & Debenhams & LDPE \\
\hline 46 & orange & Sainsbury's & HDPE \\
\hline 47 & White/green & ASDA & HDPE \\
\hline
\end{tabular}




\begin{tabular}{|c|c|c|c|}
\hline Plastic bag number & Colour & Brand & Plastic type \\
\hline 48 & Transparent & Tesco & HDPE \\
\hline 49 & White & Tesco & HDPE \\
\hline 50 & Blue & $\mathrm{N} / \mathrm{A}$ & HDPE \\
\hline 51 & Cream/black & Waterstones & LDPE \\
\hline 52 & transparent & Tesco & HDPE \\
\hline 53 & White & SAAC & LDPE \\
\hline 54 & white & NISA & HDPE \\
\hline 55 & Blue & NEXT & LDPE \\
\hline 56 & white & $\mathrm{N} / \mathrm{A}$ & HDPE \\
\hline 57 & Dark Green & $M \& S$ & LDPE \\
\hline 58 & white & New Look & LDPE \\
\hline 59 & white/green & ASDA & HDPE \\
\hline 60 & orange & Sainsbury's & HDPE \\
\hline 61 & white/red & office club & LDPE \\
\hline 62 & transparent & Tesco & HDPE \\
\hline 63 & transparent & Clarks & LDPE \\
\hline 64 & White/green & ASDA & HDPE \\
\hline 65 & white & JL & LDPE \\
\hline 66 & transparent & Tesco & HDPE \\
\hline 67 & orange & Sainsbury's & HDPE \\
\hline 68 & white & N/A & HDPE \\
\hline 69 & purple/black & National Gallery Scotland & LDPE \\
\hline 70 & white & N/A & HDPE \\
\hline 71 & black/pink & accesorise & LDPE \\
\hline 72 & white & Tesco & HDPE \\
\hline 73 & transparent & Tesco & HDPE \\
\hline 74 & red/yellow & Mozart Kugel & Cellulose \\
\hline 75 & white/black & bodycare & HDPE \\
\hline 76 & grey/cream & Next & LDPE \\
\hline 77 & white/green & ASDA & HDPE \\
\hline 78 & bronze/brown & Greece Tourist bag & LDPE \\
\hline 79 & transparent & Tesco & HDPE \\
\hline 80 & white & Liberty Duty Free & LDPE \\
\hline 81 & white/blue & WHSmith & HDPE \\
\hline 82 & Green & Fenwick Newcastle & LDPE \\
\hline 83 & transparent/Green & ASDA & HDPE \\
\hline 84 & pink/black & accesorise & LDPE \\
\hline 85 & transparent/Green & ASDA & HDPE \\
\hline 86 & transparent & Tesco & HDPE \\
\hline 87 & white/black & waterstones & LDPE \\
\hline 88 & white/blue & Boots & HDPE \\
\hline 89 & yellow/red & $H \& M$ & LDPE \\
\hline 90 & transparent/Green & ASDA & HDPE \\
\hline 91 & transparent/black & TEMT & LDPE \\
\hline 92 & white/orange & Clintons & HDPE \\
\hline 93 & white/purple & game & HDPE \\
\hline 94 & green/blue & card factory & HDPE \\
\hline 95 & white & N/A & HDPE \\
\hline 96 & cream/black & Waterstones & LDPE \\
\hline 97 & white/red & Home Bargains & HDPE \\
\hline 98 & transparent/Green & ASDA & HDPE \\
\hline 99 & White & Sports Direct & HDPE \\
\hline 100 & transparent/blue & Trespass & LDPE \\
\hline
\end{tabular}

- HDPE - high density polyethylene; LDPE - low density polyethylene 
Evaluation of the number and quality of latent marks recovered by each process

Figure 3 demonstrates that the three techniques employed in this study detected a similar number of fingermarks where superglue and BY40 detected 305 marks (of which 23 could only be detected by fluorescence), Lumicyano detected 296 marks (of which 26 could only be detected by fluorescence) and powder suspension detected 297 marks. Both light sources used in this study detected the same number of marks after treatment with Lumicyano. All three techniques yielded a small percentage $(<5 \%)$ of marks with poor contrast (grading b). For the cyanoacrylate techniques, fluorescence removed the poor contrast issues and marks could then be graded as 'a'. Although most marks could be seen visually, the use of fluorescence provided a faster visualisation method with less stress on the eye. All three techniques were capable of developing marks with third level ridge detail. Over-fuming of marks was rarely observed with both cyanoacrylate techniques. Subsequent treatment of Lumicyano-enhanced marks with BY40 detected an additional 43 marks (figure 3).

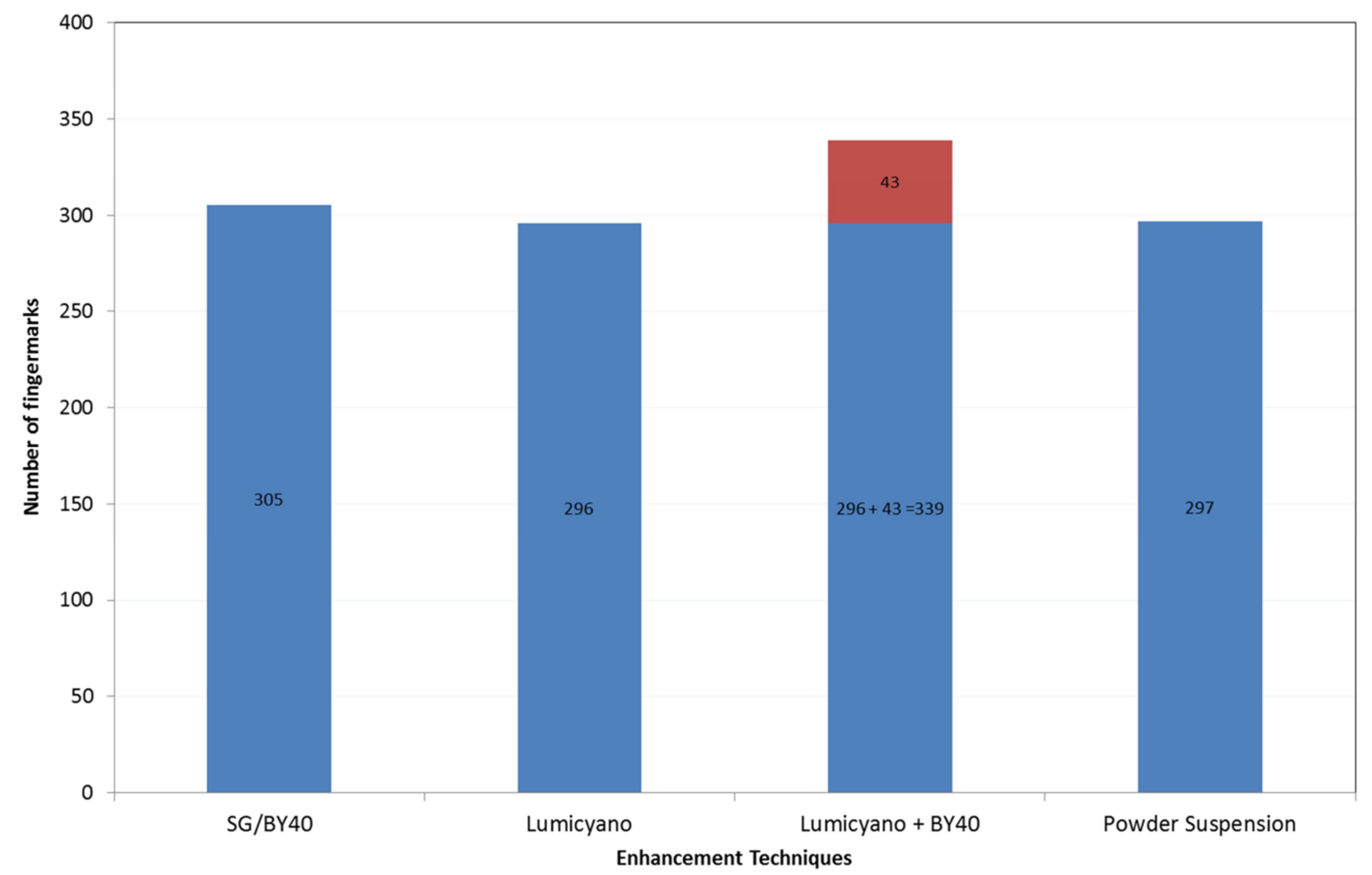

Figure 3 - The number of enhanced latent fingermarks for each process 
There were some differences between each technique although all techniques detected a similar number of marks. In general, Lumicyano proved to be an effective technique as long as the manufacturer's guidelines are followed. This mainly refers to having the product at room temperature after removing from cold storage and that the bottled product is mixed by shaking for at least 45 seconds prior to use. Furthermore, the fuming cabinet must be clean prior to use as Lumicyano glue is attracted to old cyanoacrylate residues. In comparison to the other two techniques, Lumicyano did not require any dyeing or drying facilities/times thus saving time and lab space. Both the UV light and the Quaser used in this study found the same number of marks; however, in general, the blue/green light and orange filter combination (Quaser) provided better contrast, specifically on white and highly reflective backgrounds (figure 4).

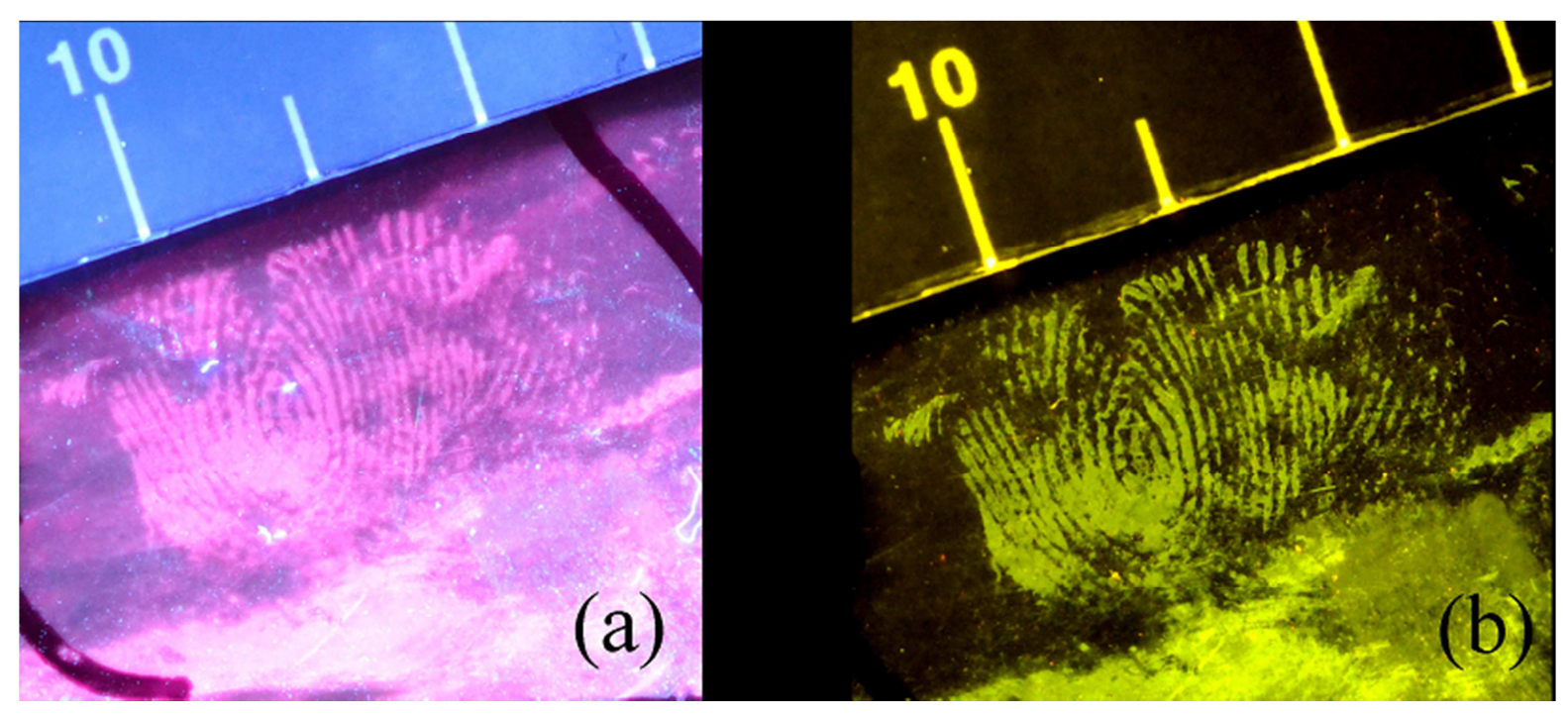

Figure 4 - A latent mark on a carrier bag after treatment with Lumicyano observed with (a) UV light and (b) blue/green light (orange filter)

The use of traditional superglue followed by BY40 proved to be an effective enhancement technique but required dyeing and rinsing facilities as well as a drying area. On the plus side, observation of marks treated with BY40 provided very strong fluorescence that did not degrade by exposure to light (figure 5). The use of powder suspensions was also an effective enhancement technique (figure 6) but requires a large sink and drying area for batch processing. In addition, when treating one side of the bag, marks on the other side might be destroyed in the process. Nonetheless, it can be argued that it is more likely to detect marks on the outside, rather than the inside, of the bag and that the outside should be treated first, dried and analysed before treating the inner side. Both powder suspension and superglue/BY40 required a drying time of at least 2-3 hours before examination. 


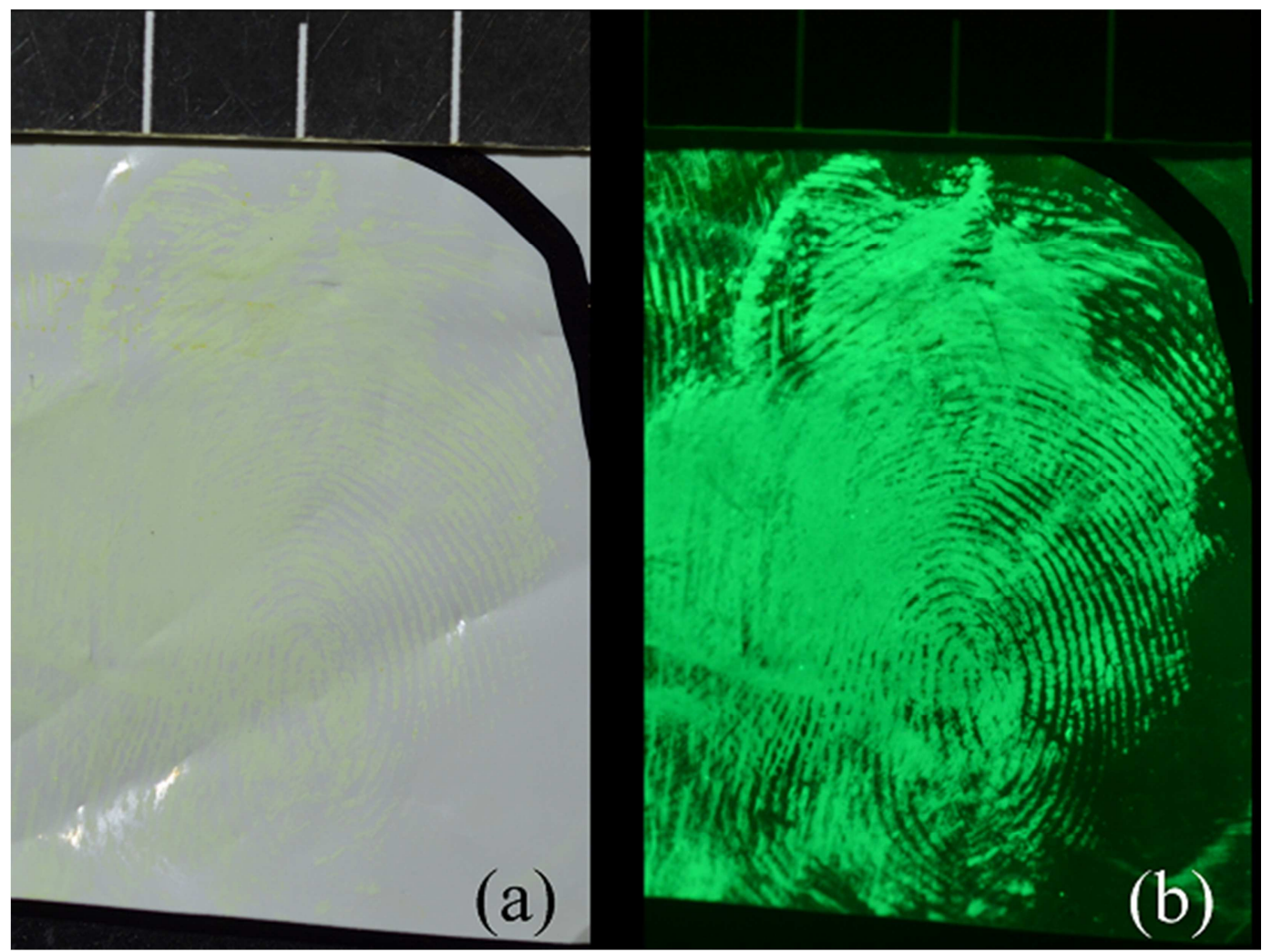

Figure 5 - A latent mark on a carrier bag after treatment with superglue and BY40 under (a) white light and (b) violet/blue light (yellow filter)

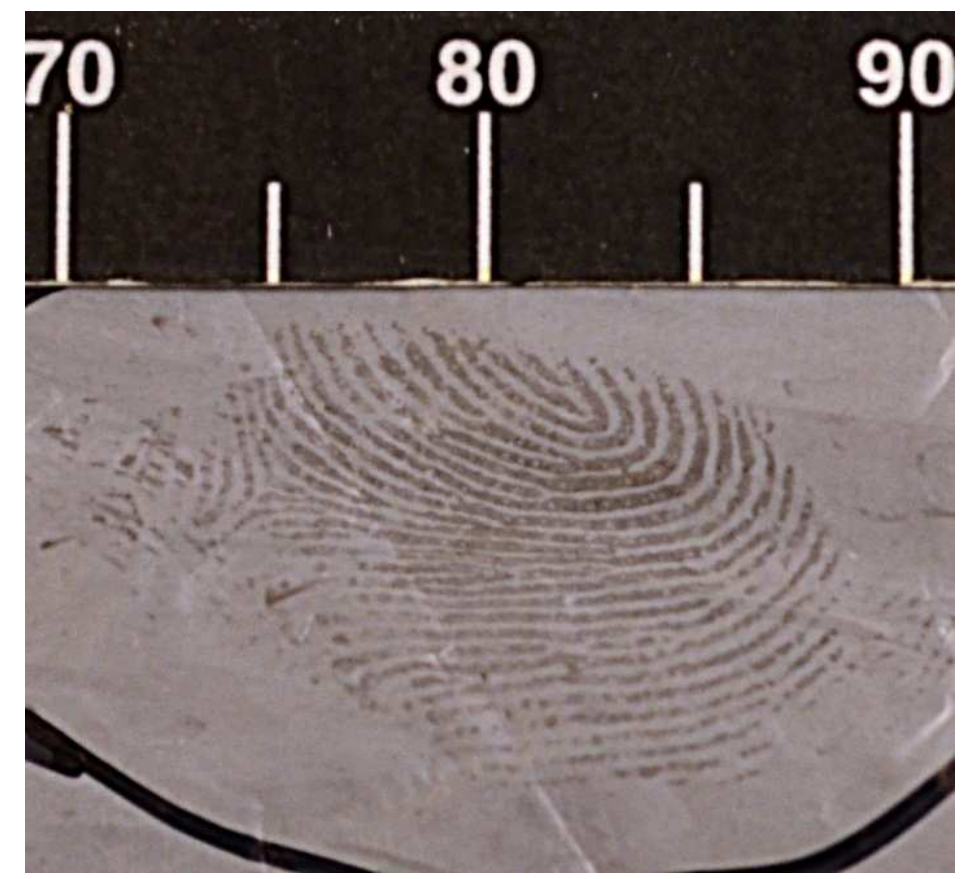

Figure 6 - A latent mark on a carrier bag after treatment with black powder suspension 


\section{Evaluation of the stability of Lumicyano fluorescence}

A selection of fingermarks developed with Lumicyano $(\sqrt{ } 296 \sim 17)$ was investigated further for the stability of fluorescence. The manufacturer's guidelines state that examination and photography should take place within 48 hours of treatment in order to ensure the quality of the fluorescence. In this study, when the halved latent fingermarks treated with Lumicyano were stored under daylight conditions, the fluorescence deteriorated after 1 day, to the extent that it was a strain on the operator's eye and could potentially be missed (figure 7c). When these marks were examined after 1 week, no fluorescence was observed. For the halved Lumicyano treated marks that were stored in the dark, the deterioration of fluorescence was much slower and was detectable after 1 week (figure 7d). Further trials on marks treated with Lumicyano that were stored in the dark demonstrated that observation of fluorescence is still possible after a period of six months. Additionally, these trials demonstrated that the fluorescence of the Lumicyano processed marks decreased over time depending on the environmental conditions, such as humidity and temperature, as well as the substrate. It was also possible to restore or strengthen the fluorescence by re-fuming with Lumicyano (figure 7e); however, it was not always as bright as the 1 hour samples (figure 7b). It was also possible to treat the re-fumed marks with BY40 (figure 7f). Manipulation with computer software of the acquired images is likely to enhance the fluorescence in figure 7 further. None of the images presented in this study have been enhanced with computer software to improve fluorescence. 


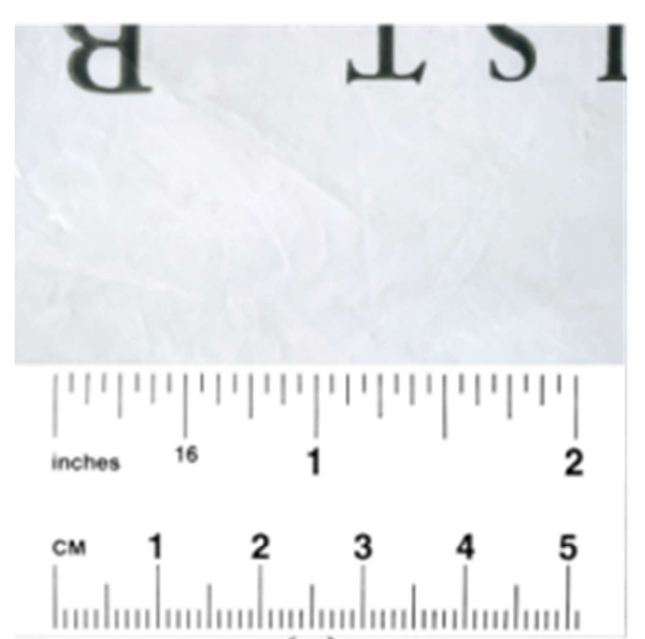

(a)

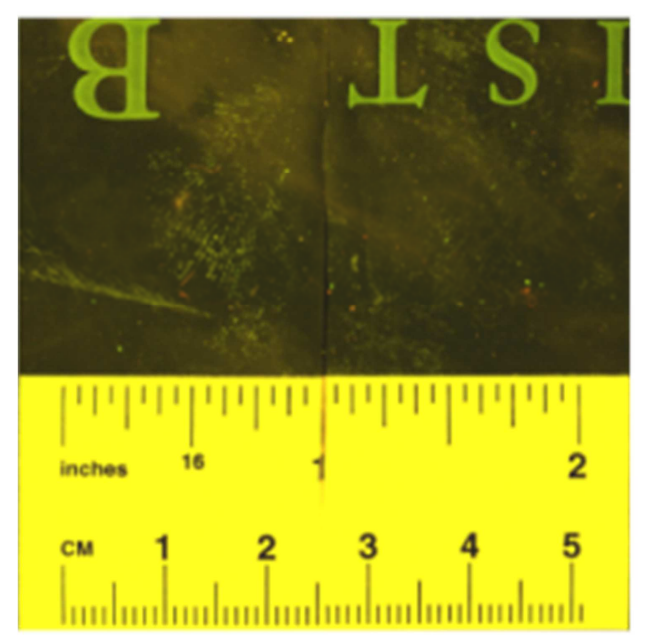

(c)

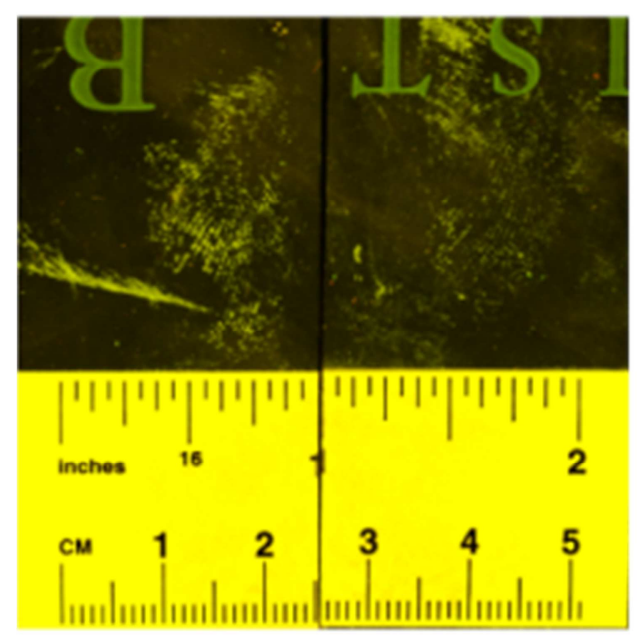

(e)

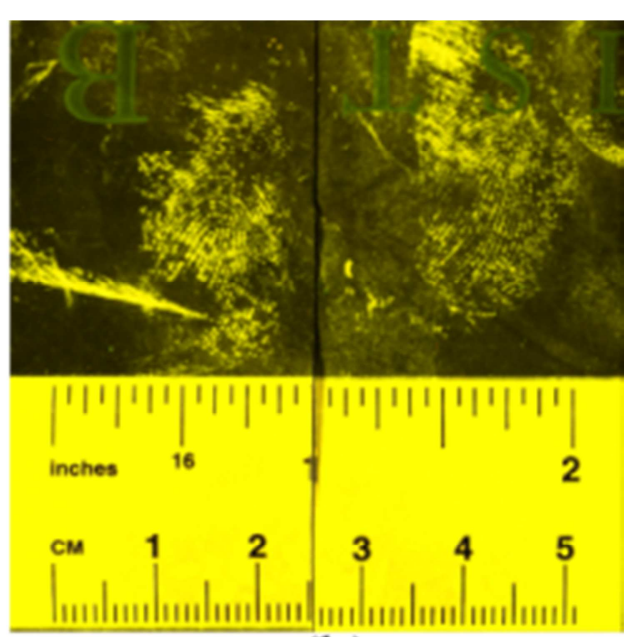

(b)

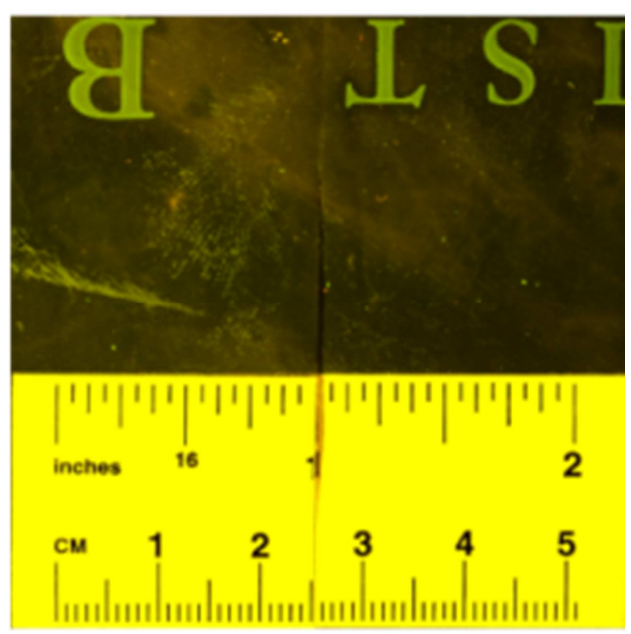

(d)

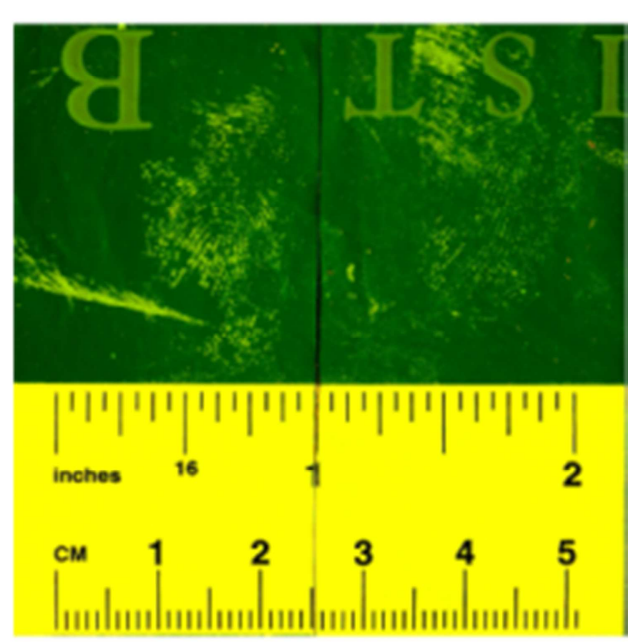

(f)

Figure 6 - A fingermark on a plastic carrier bag treated with Lumicyano [left part stored in the dark, right part stored on open bench] under (a) white light (b) blue/green (BG) light (orange filter) within an hour of fuming (c) BG light (orange filter) after 1 day (d) BG light (orange filer) after 1 week followed by (e) re-fuming with Lumicyano after 1 week [BG light (orange filter)] and (f) sequential BY40 treatment of (e) [violet/blue light (yellow filter)] 


\section{Conclusion}

The use of a new superglue product, Lumicyano, provides a comparable fingermark detection rate to superglue/BY40 and powder suspension. The use of Lumicyano provides a one-step fuming and dyeing treatment in a superglue chamber that does not require any modifications and thus avoiding health and safety issues arising from the heating of cyanoacrylate at high temperatures. In comparison to the other two techniques, Lumicyano does not require any dyeing or drying facilities/times thus saving time and lab space. After treatment with Lumicyano, it is recommended to perform fluorescence examination immediately. If this is not possible, the fumed articles should be stored in a cool, dark and dry place, ideally sealed in a Kraft envelope to prevent any air circulation and checked for fluorescence at the earliest opportunity. Nevertheless Lumicyano process provides an excellent signal to noise ratio and digital processing may improve the intensity of the signal. Further treatment with BY40 for Lumicyano-enhanced marks may detect additional marks and provide brighter fluorescence that does not degrade on exposure to daylight. Further research will assess the use of Lumicyano on other surfaces, under vacuum conditions and the use of other light sources for brighter fluorescence. 


\section{References}

1. V. Bowman (Ed.), Manual of Fingerprint Development Techniques, 2nd ed., $3^{\text {rd }}$ rev, Home Office Scientific Development Branch, Sandridge, UK, 2005.

2. R. Downham, S. Mehmet, V.G. Sears, A Pseudo-Operational Investigation into the Development of Latent Fingerprints on Flexible Plastic Packaging Films, Journal of Forensic Identification 62 (6) (2012) 661-682

3. Lumicyano Global Forensics, September 2013 [Internet]. Available from: http:// www.globalforensics.co.uk/lumicyano.htm

4. W. Hahn, R.S. Ramotowski, Evaluation of a novel one-step fluorescent cyanoacrylate fuming process for latent print visualisation, Journal of Forensic Identification, 62 (3) (2012) 279-298

5. T.C. Fung, K. Grimwood, R. Shimmon, X. Spindler, P. Maynard, C. Lennard, C. Roux, Investigation of hydrogen cyanide generation from the cyanoacrylate fuming process used for latent fingermark detection, Forensic Science International, 212 (1-3) (2011) 143-149

6. C.A. Steele, M.A. Hines, L. Rutherford, Specific Heat Capacity Thermal Function of the Cyanoacrylate Fingerprint Development, NIJ report, Document number: 238263, Award number: 2009-DN-BX-K196 (2012)

7. V.G. Sears, S.M. Bleay, H.L. Bandey, V.J. Bowman, A methodology for fingermark research, Science \& Justice, 52 (3) (2012) 145-160 